\title{
Conquering carbohydrate complexity
}

Despite mass spectrometry's success in the identification and characterization of small molecules (Milestone 7), its application to carbohydrates was initially stymied. This was due to their low vapor pressure-which made it difficult to push them into the gas phase-and heat sensitivity, which led to low reproducibility or poor signal intensity. In 1959, however, P.A. Finan and R.I. Reed published a spectrum of a methylated polysaccharide, suggesting that carbohydrate modifications could solve the problem of low vapor pressure.

On the basis of this technical clue, Klaus Biemann and colleagues and N.K. Kochetkov and colleagues followed up with two comprehensive reports in 1963, presenting the mass spectra of a variety of methylated or acetylated monosaccharides and establishing mass spectrometry as a viable technique for the structural determination of carbohydrates. The two groups identified characteristic patterns of ions that reflect the chemical cleavage of the monosaccharides into different fragments. In tandem with the spectra obtained from deuterated monosaccharides to monitor specific portions of the ring structure, these patterns can be used to identify the ring size and position of some functional groups.

Over the next two decades, additional reports emerged that focused on longer carbohydrate sequences or specific types of carbohydrates; inefficiencies in the methylation reactions, however, remained a roadblock. In a seminal report in 1984, lonel Ciucanu and Francisc Kerek revisited the chemical mechanism of this methylation reaction, discovering that simple bases such as $\mathrm{OH}^{-}$or $\mathrm{H}^{-}$ were sufficient to catalyze the reaction. Their revised reaction conditions improved yields from $30 \%$ to $100 \%$, shortened the reaction time from hours to minutes, eliminated by-products that had plagued earlier techniques and used chemicals that were more accessible to researchers.

With improved techniques in hand, and the development of tandem mass spectrometry (Milestone 13), scientists in the field were poised to investigate more complex carbohydrates. However, it quickly became clear that the nomenclature developed to describe carbohydrate fragments was insufficient to capture this new information. In 1988, Bruno Domon and Catherine Costello defined a new systematic nomenclature, based on recently developed peptide terminology, that indicated where chemical cleavage occurred within a saccharide structure, which 'end' of the carbohydrate structure the observed fragment was from and whether the fragment was part of a branch or the 'core' structure.

From the 1990s to early 2000s, the dissemination of commercial electrospray mass spectrometers, along with other improvements in instrumentation, provided major advances in carbohydrate analysis. Douglas Sheeley and Vernon Reinhold demonstrated that the quadrupole ion trap mass spectrometer was superior to earlier instruments because of its efficiency in creating and isolating ions. As a result, it enabled multiple tandem mass spectrometry experiments and detection of less abundant fragments, facilitating more complete assignments of the connections between substructures than had been possible before.

Whereas previous studies on glycopeptides had examined only the isolated peptide or glycan structure, Alan Marshall and Carol Nilsson and colleagues reported the use of a single instrument-the Fourier transform ion cyclotron resonance mass spectrometer (see Milestone 5) - to collect two different kinds of spectra of intact glycopeptides. By using the 'soft' fragmentation technique of electron-capture dissociation, the researchers were able to detect fragments of the peptide backbone, whereas infrared multiphoton dissociation reported on the carbohydrate structure, facilitating glycopeptide assignment.

This combination of methods for modification, characterization and interpretation remains the standard today, powering increasingly insightful explorations of the glycome.

Catherine Goodman, Senior Editor, Nature Chemical Biology

\footnotetext{
ORIGINAL RESEARCH PAPERS Biemann, K., DeJongh, D.C. \& Schnoes, H.K. Application of mass spectrometry to structure problems. XIII. Acetates of pentoses and hexoses. J. Am. Chem. Soc. 85, 1763-1771 (1963) | Kochetkov, N.K., Wulfson, N.S., Chizhov, O.S. \& Zolotarev, B.M. Mass spectrometry of carbohydrate derivatives. Tetrahedron 19, 2209-2224 (1963)| Ciucanu, I. \& Kerek, F. A simple and rapid method for the permethylation of carbohydrates. Carbohydr. Res. 131, 209-217 (1984) | Domon, B. \& Costello, C.E. A systematic nomenclatur for carbohydrate fragmentations in FAB-MS/MS spectra of glycoconjugates. Glycoconjugate J. 5, 397-409 (1988) Sheeley, D.M. \& Reinhold, V.N. Structural characterization of carbohydrate sequence, linkage and branching in a quadrupole ion trap mass spectrometer: neutral oligosaccharides and $\mathrm{N}$-linked glycans. Anal. Chem. 70, 3053-3059 (1998)| Håkansson, K. et al. Electron capture dissociation and infrared multiphoton dissociation MS/MS of an N-glycosylated tryptic peptide to yield complementary sequence information. Anal. Chem. 73, 4530-4536 (2001) FURTHER READING Finan, P.A. \& Reed, R.I. Application of the mass spectrometer to polysaccharide chemistry. Nature $\mathbf{1 8 4}$ 1866 (1959) | Hirabayashi, J., Arata, Y. \& Kasai, K. Glycome project: concept, strategy and preliminary application to Caenorhabditis elegans. Proteomics 1, 295-303 (2001)
} 